\title{
Diversidad Sorda: educación y sensibilidad intercultural en una escuela especial de Santiago de Chile
}

\author{
Deaf Diversity: intercultural sensitivity education of a special \\ school in Santiago of Chile
}
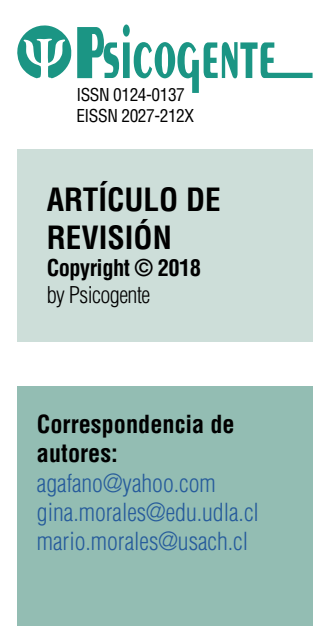

Recibido: $15-02-17$ Aceptado: 05-05-17 Publicado: $01-07-18$

\begin{tabular}{|c|}
\hline $\begin{array}{l}\text { Gina Viviana Morales Acosta iD } \\
\text { Universidad de Antofagasta. Facultad de Ciencias de la Salud. Departamento de Cs. de la } \\
\text { Rehabilitación y Movimiento Humano. Santiago de Chile, Chile }\end{array}$ \\
\hline $\begin{array}{l}\text { Mario Morales Navarro iD } \\
\text { Universidad Santiago de Chile. Santiago de Chile, Chile }\end{array}$ \\
\hline
\end{tabular}

\section{RESUMEN}

La finalidad del presente artículo consiste en describir los componentes de educación y sensibilidad intercultural que se evidencian en la práctica docente con el estudiante sordo en la escuela especial. Se destaca el principio sobre estudiantes sordos estipulado en el documento de la UNESCO (2006) en pro de los aprendizajes en educación, que debe asumir los deberes y derechos inherentes al sujeto sordo en su particularidad lingüística: "La educación intercultural respeta la identidad cultural del educando impartiendo a todos una educación de calidad que se adecúe y adapte a su cultura". De acuerdo con ello, los principios de educación intercultural deben construirse considerando que: I) Se respete la identidad cultural del educando impartiendo a todos [as] una educación de calidad que se adecúe y se adapte a su cultura; II) Se enseñe a cada educando los conocimientos, las actitudes y las competencias culturales necesarias para que pueda participar plena y activamente en la sociedad; III) La elaboración de programas propios de estudio. Esto exige que la institución promueva el acercamiento a la asociación y a la comunidad sorda, para que los estudiantes la conozcan e interactúen en etapas tempranas y así propendan hacia la negociación simétrica de las culturas para reconocer a un Otro diverso en las prácticas docentes del aula.

Palabras Clave: sensibilidad intercultural, diversidad sorda, práctica docente, escuela especial, educación intercultural.

Abstract

This paper aims to describe the components on intercultural sensitivity education, evident in teaching process to deaf learners of a "special school". To respect deaf learners' cultural identity through high quality education adapted to their culture, is the principle of this intercultural education, as per (UNESCO, 2006) for Deaf students, in favor of learning-education, which must assume duties and rights related to their linguistic features, inherent to the deaf learner. Intercultural education principles are based on I) To respect deaf learner cultural identity, not only giving them a high-quality education but also adapted to their culture. II) To teach deaf learners: necessary knowledge, attitudes and cultural skills to participate fully and actively in society. III) To develop study programs by the special school; these programs include : as a contribution, a theoretical revision, based on the construction of sensibility patterns to develop the intercultural education principles actively. Also, it implies that the "special school" must promotes the approaching to the association and the Deaf community, so that means, the students are able to know it and interact with other deaf student in early stages in order to recognize a diverse Other, tending towards the symmetrical negotiation of the cultures in teaching practices, conducted through communicative process in the classroom.

Keywords: intercultural sensitivity, deaf diversity, teaching practice, special school, intercultural education.

Cómo citar este artículo (APA):

Morales Acosta, G. V. \& Morales Navarro, M. (2018). Diversidad Sorda: educación y sensibilidad intercultural en una escuela especial de Santiago de Chile.

Psicogente 21(40), 458-475. https://doi.org/10.17081/psico.21.40.3083 


\section{INTRODUCCIÓN}

\subsection{Escuela y estudiantes sordos}

Como punto de inicio, en este trabajo se considera importante resaltar la escritura de la palabra Sordo (con inicial mayúscula) (Woodward, 1972), develando con ello la enunciación y posicionamiento comunicativo en la reivindicación de la lengua de señas, y respeto a la cultura Sorda que guía este estudio y, por ende, al lector.

De este modo se reconoce, además, que dentro de la comunidad Sorda se vive la diversidad inherente a la condición humana. Al respecto, Morales et al., (2017) plantean que la diversidad auditiva se daría, desde una primera vertiente, según el grado de audición, que contaría con aditamentos o ayudas técnicas (audífonos) que no son excluyentes para el aprendizaje de la lengua de señas; en tanto que, en una segunda vertiente, una diversidad Sorda usuaria de la lengua de señas, como también con otros métodos comunicativos como los aprendidos en terapias como el oral y el bimodal o que alternan la lengua de señas y la oralidad.

Los lineamientos internacionales y nacionales se encuentran permeados, en primer lugar, por el énfasis puesto en el diagnóstico audiológico, con un abordaje asumido de forma individual y a partir de un modelo médico de pérdida. Acorde con esto, la información inicial que recibe la familia se orienta hacia ayudas técnicas: audífono o implante coclear, ajena a una guía o acompañamiento comunicativo que permita la adquisición y desarrollo de la lengua de señas del país de origen en una edad temprana. En este sentido, cabe mencionar que la lengua de señas no es universal, y más bien posee una gramática propia y estructuras sintácticas inherentes a una lengua en constante desarrollo (Adamo et al., 2009).

Lo anterior se refleja en la educación del Sordo y devela desde qué punto de enunciación comunicativa se han posicionado históricamente directivas y docentes como sujetos situados (Zemelman, 2010, 1998, 1992) para el abordaje pedagógico del problema en la escuela: a) en primer lugar, desde un modelo auditivo-médico, con mirada hacia el oralismo, que en Chile se ve reflejado en los métodos comunicativos utilizados en la educación de los Sordos, en los cuales convergen formas de comunicación que pueden contener: lengua oral, bimodal, como el uso simultáneo de algunos apuntadores de lengua de señas y expresiones orales; b) en segundo lugar, un abordaje desde el modelo social, que se cimienta en el respeto a la cultura 
Sorda y en el posicionamiento de la lengua de señas como lengua materna en el uso académico, lo que tiene una estrecha relación con el acceso al conocimiento en su lengua propia.

Legalmente, Chile reconoce la lengua de señas como una lengua propia de los Sordos: en la Ley N² 20422 de febrero/2010, el art. 26 expresa: "La lengua de señas como medio de comunicación natural de la comunidad Sorda. Esta lengua se encuentra inmersa en los contextos familiares, directivos y docentes por lo que, a partir de este criterio, posicionaremos el presente estudio sobre la llamada Sensibilidad Intercultural, conceptualizada como "la capacidad de un individuo para desarrollar una emoción positiva hacia la comprensión y apreciación de las diferencias culturales que promueve un comportamiento adecuado y eficaz en la comunicación intercultural" (Chen, 1997). Y dicha Sensibilidad, fundada en el aspecto afectivo de la competencia comunicativa Intercultural, articula componentes cognitivos y conductuales (Chen y Starosta, 2000).

Las personas interculturalmente sensibles deben poseer características como: participación e implicación en la interacción, la capacidad de no juzgar, apertura mental, autoestima, supervisión, auto-concepto y empatía; todo ello para apoyar y recrear vivencias mediante el lenguaje que se enfoque hacia el aprendizaje, en donde este pasa por el otro de forma colaborativa y solidaria.

Según Mico-Cebrián \& Cava (2014), esta Sensibilidad Intercultural implica un desafío en directivas y prácticas docentes, de manera concreta en el reconocimiento y estatus de la lengua que permitirían delinear en la institución la presencia de principios de educación intercultural (Unesco, 2006), de acuerdo con los siguientes principios: I) Respeta la identidad cultural del educando, impartiendo a todos [as] una educación de calidad que se adecúe y se adapte a su cultura; II) Enseña a cada educando los conocimientos, las actitudes y las competencias culturales necesarias para que pueda participar plena y activamente en la sociedad; III) Enseña a todos los educandos los conocimientos, actitudes, y las competencias culturales que les permiten contribuir al respeto, el entendimiento y la solidaridad entre individuos, así como entre grupos étnicos, sociales, culturales, religiosos, y entre naciones.

Estos ajustes de los principios de educación intercultural permiten entender que reconocerse como escuela intercultural desde el $\mathrm{PEI}$, no necesariamente 
pasa por recursos del Estado, que exclusivamente legitima lo intercultural con pueblos originarios o pueblos indígenas.

Desde esta perspectiva, a continuación se problematiza la diversidad Sorda desde la revisión teórica, como aporte de la escuela especial en la construcción de caminos de sensibilidad para desarrollar los principios de educación intercultural de forma activa.

\subsection{LA SENSIBILIDAD INTERCULTURAL}

Antes de estudiar en forma básica la interacción y los posibles elementos de la sensibilidad de la competencia comunicativa intercultural en el aula, que propiciaría el docente en estudiante con diversidad Sorda, se presentan datos estadísticos para que el lector dimensione la realidad sobre la condición actual de la población con discapacidad.

El informe presentado por la Comisión Económica para América Latina y el Caribe señala en este sentido que: "[...] ]es posible estimar que, en la actualidad, alrededor del 12.0 \% de la población de América Latina y el Caribe viviría al menos con una discapacidad, lo que involucraría aproximadamente a 66 millones de personas" (CEPAL, 2012).

En Chile, el estudio del Servicio Nacional de Discapacidad expone un estimado del $12.7 \%$ de personas, lo que supondría un aproximado en el año 2004 de 2.2 millones y un aumento en el estudio del año 2015 a 2.6 millones de personas en situación de discapacidad; $y$ de este conjunto, en el año 2004 declaran que 488.511 corresponden a la población con pérdida auditiva. Además, según el Estudio Nacional de la Discapacidad (2015), en Chile 1.114.911 personas declaran tener dificultades para oír, equivalentes al $6.9 \%$ de la población total del país (Senadis, 2004, 2015).

Sin cuantificar los casos de personas que la adquieren como consecuencia del proceso de envejecimiento normal o, sin ser menos importante, por la exposición al ruido como causa o consecuencia del trabajo, que puede originar hipoacusia inducida por ruido o hipoacusia laboral; cabe mencionar que la hipoacusia puede ser una patología de nacimiento o adquirida por traumas craneoencefálicos, traumas acústicos o medicamentos ototóxicos.

Entre los anteriores datos estadísticos, se halla que el $5 \%$ de los Sordos tiene padres Sordos ${ }^{1} y$, por tanto, mayores posibilidades de acceso a una lengua

1 CODA (Children of Deaf Adult- CODA por sus siglas en inglés) significa Hijos de Padres Sordos, en donde el 90-95\% de los hijos de adultos Sordos son oyentes (Schein, 1989, citado en Hadjikakou et al., 2009). 
materna temprana; mientras que el otro $95 \%$ tiene padres oyentes, lo que da cabida a un abanico de posibilidades bastante variable que inicia al momento del nacimiento de los hijos, cuando los padres vivencian o no diferentes niveles de manejo de la lengua de señas para acompañar el desarrollo del lenguaje de su hijo (Massone et al., 2012).

El Gobierno de Chile no cuenta con políticas lingüísticas para la primera infancia Sorda, lingüísticamente considerada como minoría (UNESCO, 2010) en forma paralela al diagnóstico médico, como tampoco con un acompañamiento a la familia por parte de Sordos adultos como modelos lingüísticos y profesionales de la educación. En estas circunstancias, para evitar un retraso en la adquisición y desarrollo del lenguaje de una manera posterior y no simultánea al diagnóstico, la familia inicia de forma individual y asimétrica el proceso de adquisición y desarrollo del lenguaje del hijo Sordo, en terapias de lectura labial para que el hijo desarrolle pensamiento complejo.

La privación del lenguaje repercute en el desarrollo intelectual del infante, y de manera concreta en los aspectos conductual, cognitivo, psicológico y social, si no accede a una lengua natural, ya sea oral o de señas, que le sea efectiva para realizar una representación del mundo. En el acceso temprano o posterior a la lengua de señas, se encuentran niveles distintos de competencia lingüística en la comunidad Sorda; y desde su quehacer, el intérprete sitúa los problemas intrínsecos, límites o posibilidades de la interacción comunicativa, para ejemplificar, explicar y unificar señas que proporcionen una mejor claridad sobre el tema.

El proceso de adquisición de la Lengua de Señas debería iniciarse de forma temprana, con los procesos de significación, pues, a medida que el niño crece, se enfrenta a la experiencia del lenguaje, de modo que, si no tiene una lengua materna, tendrá un repertorio lingüístico reducido que no le permitirá comunicarse ampliamente con un posible interlocutor.

La anterior afirmación se fundamenta en dos aspectos teóricos complementarios: El primero hace referencia a los mecanismos básicos de la plasticidad cerebral, donde el sistema nervioso tiene una mayor capacidad de aprendizaje a nivel funcional. Ello permite a los niños Sordos apropiarse de la Lengua de Señas como primera lengua en una etapa temprana y, a la vez, les posibilita un mejor dominio en todos los niveles del lenguaje. Así se evita que el niño, y posteriormente el adulto, haga uso de códigos señados restringidos 
o caseros, que solo son comprendidos por la familia o el círculo social más inmediato.

El segundo aspecto comprende la participación en entornos lingüísticamente ricos y contextos representativos, lo que le permitirá a niñas y niños Sordos un desempeño acorde con las exigencias sociales y tecnológicas. Esto es necesario en tanto "los niños Sordos deben de tener acceso a la construcción lingüística de la misma manera que un niño oyente" inmerso en el llamado "baño del lenguaje". Pero, para que el niño Sordo pueda beneficiarse de una verdadera estimulación lingüística, su entorno social ha de estar constituido por locutores de lengua de señas, elemento importante para la construcción de una positiva autoimagen y una identidad.

En algunas ocasiones es necesario hacer "adecuaciones" a dichos entornos, propiciando, por ejemplo, el contacto con modelos lingüísticos, co-docentes y adultos que favorezcan la adquisición natural de la lengua de señas, de modo que el discurso en lengua de señas fluya en tipologías conversacionales, explicativas, expositivas y narrativas, en el proceso de desarrollo normal del lenguaje que se da en los primeros años y teniendo en cuenta que los niños tienen sus propias hipótesis del mundo y de la forma como suceden los fenómenos mediante hipótesis que les son útiles como base para afirmarlas o refutarlas. En esta medida, necesitan un código que les permita concretarlas y darlas a conocer para modificarlas. $Y$ es que la situación comunicativa comprendida en el ingreso a la escuela se ve reflejada en la interacción, que debe ser considerada desde los principios de educación intercultural (UNESCO, 2006).

Dicha sensibilidad intercultural implica un desafío para directivos, docentes y codocentes en relación con sus prácticas educativas y pedagógicas respecto al reconocimiento y estatus de la lengua de señas en el aula. Ya que el estudiante, a su ingreso, no puede acceder de forma plena al conocimiento si no aprende antes a comunicarse. Situación que devela la necesidad de realizar adecuaciones visuales para el contexto pedagógico en construcción, que permitan frenar situaciones adversas a estudiantes Sordos y ejecutar los lineamientos de principios de educación intercultural.

En este espacio vital, la escuela de la presente revisión debe adscribirse desde un PEI bilingüe intercultural ${ }^{2}$, cuestión que, más allá de relaciones étnicas,

2 Es de importancia señalar que el concepto bilingüe para Sordos en este estudio será planteado con el objetivo de fortalecer la lengua materna (L1) como la Lengua de Señas Chilena y los componentes que se complementan y confrontan, en el enfoque teórico-conceptual, desde una aproximación de educación intercultural en Chile y sensibilidad de las competencias comunicativas interculturales que subyacen dentro del aula. 
supone la transversalidad, en la lengua de señas, de la comunicación de Sordos afrodescendientes, indígenas y mestizos. Es aquí donde el Ministerio de Educación enfrenta el desafío para articularse a la escuela especial, es decir, a los programas de Educación Intercultural y bilingüe en Chile, que propicie recursos, planes y programas propios, en simetría, en la mediación comunicativa que se expresa de forma "artificial" ${ }^{3}$ en el espacio escolar.

La educación intercultural en y con diversidad Sorda, que si bien en sus orígenes se ha direccionado principalmente (por no decir que en forma única) a los pueblos originarios, requiere una propuesta que contemple la interculturalidad en Sordedad (Ladd, 2011), no tanto como derivación de pueblos originarios, sino como representación de la interacción en competencia comunicativa intercultural, es decir, con planes y programas visuales propios, que propicien dinámicas simétricas de formas consensuadas y que se reflejen dentro del aula en la interacción docente/estudiante.

En los lineamientos para séptimo a segundo medio, en el desarrollo de la competencia comunicativa oral (comprensión, interacción y expresión), se lee:

Teniendo esto en consideración, los propósitos apuntan a la adquisición de competencias comunicativas necesarias para el desarrollo integral de los estudiantes. Dado que estas competencias solo se alcanzan con el uso, se hace primordial proveer a los alumnos de variadas oportunidades para hablar, escuchar, leer, observar y escribir, y así darle significado al mundo que los rodea y prepararlos para su futura participación en todos los ámbitos de la sociedad. (MINEDUC, 2012, p.25)

Desde esta normativa, las interacciones que se dan en educación especial han sido pensadas en su génesis por oyentes para Sordos, entendiéndose las competencias, delineadas por el Ministerio de Educación, desde el currículo nacional: “(...) de manera que accedan, participen y progresen en igualdad de condiciones y oportunidades" (MINEDUC, 2009).

King y Baxter (2005) aducen que la competencia comunicativa permite al docente/estudiante hacerse más competente mediante la interacción con otros grupos culturales, a través de la experiencia y la decisión consensuada de normas. En la dimensión emocional de esta competencia, se tendrán en

3 Artificial porque los estudiantes con diversidad Sorda deben aprender lengua de señas a través de aditamentos pedagógicos, y algunos no vienen con ese bagaje comunicativo desde el hogar, que permita de forma fluida acceder a otras esferas del conocimiento. De forma paralela, la escuela debe enseñar conocimiento y formas de comunicarse. 
cuenta componentes ${ }^{4}$ de sensibilidad intercultural (Chen y Starosta, 1998) como: implicación en la interacción, respeto ante las diferencias culturales, confianza, grado en que se disfruta y atención en la interacción.

El mismo Chen (1997) define el concepto de Sensibilidad Intercultural como nuestra capacidad para "desarrollar una emoción positiva hacia la comprensión y apreciación de las diferencias culturales que promueven un comportamiento adecuado y eficaz en la comunicación intercultural" (p.5). Más en concreto, se han especificado seis elementos a tener en cuenta en la sensibilidad intercultural: Autoestima, auto-monitoreo, apertura mental, empatía, involucrarse en la interacción y el no juzgamiento (no censura).

Los autores involucran tres conceptos al referirse al tema: a) la conciencia intercultural, b) la competencia comunicativa intercultural, de las cuales forma parte lo cognitivo, lo comportamental y lo afectivo, y c) la sensibilidad intercultural. Estos conceptos se explican en la Gráfica 1.

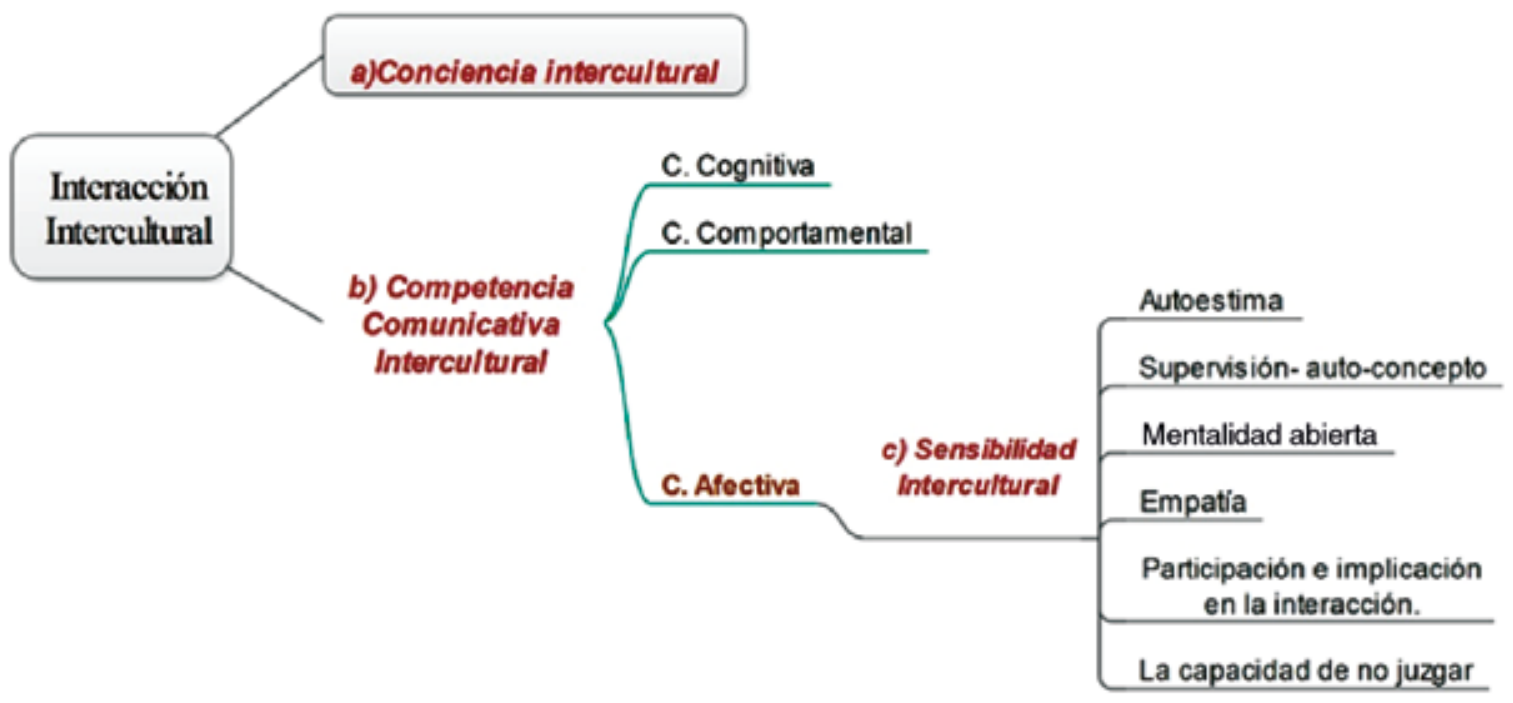

Gráfica 1. Conceptos de Sensibilidad intercultural (Chen, 1997)

Las dimensiones de la competencia comunicativa intercultural son: competencia cognitiva, comportamental y afectiva (Chen y Starosta, 1998). Este trabajo se enfoca en la última de estas dimensiones. En función del respeto de las interacciones, busca alternativas que incluyan una construcción de manera conjunta en el desarrollo de una interacción como: autoestima, autocontrol, abierto a nuevas perspectivas/apertura mental, la empatía, la

4 En este trabajo en particular se alude a componentes como indicadores en la teoría validity or a new instrument, the intercultural Sensitivity Scale (ISS) (Chen \& Starosta, 2000) 
participación de la interacción y el evitar la emisión de un juicio de valor. De hecho, uno de los componentes que enriquece las interacciones en la Competencia Comunicativa Intercultural consiste en utilizar proactivamente y conocer profusamente una lengua, en este caso la lengua de señas, en conjunto con las ayudas técnicas, en diada intercultural, con elementos esenciales como son: empatía, criterios de cooperación, expresión y disposición a ampliar y reconocer las señas nuevas.

La sensibilidad de la competencia comunicativa intercultural para estudiantes Sordos incluye componentes visuales, elementos que ingresan en la adquisición y desarrollo del lenguaje para la comunicación en forma gradual, como también las interacciones culturales. Se puede decir, sin embargo, que una persona Sorda u oyente con manejo de lengua de señas tiene competencia comunicativa para situaciones cotidianas o de baja complejidad, pero que no logra acceder a situaciones de media y alta complejidad, por no contar con elementos que le permitan sostener una conversación en el área de una forma coherente y cohesiva; ello equivale, por ejemplo, a conocer solo un par de palabras del español y, sin embargo, pretender dar una clase en ese idioma. Así, la selección de señas [palabras] del lenguaje científico o pedagógico es uno de los desafíos de las prácticas docentes para construir conceptos nuevos en áreas del conocimiento.

El carácter cambiante de la sociedad supone reconocer el aporte de la cultura de un Otro diverso, el respeto a lo desconocido y el aprender a trabajar y vivir juntos, con y en las diferencias que las personas manifiesten en las interacciones cotidianas. Lo anterior exige de la escuela una fuerte demanda, y en especial por parte de los docentes, en el sentido de lograr una mayor comprensión, sensibilidad y competencia entre una cultura Sorda y cultura Oyente. Imaginemos por un momento ¿qué pasa cuando un sujeto Sordo, que ha desarrollado su facultad del lenguaje y en esta ha construido un mundo de sentidos en imágenes de la lengua de señas, se quiere comunicar con otro sujeto que se mueve con significaciones basadas en la expresión oral? Justo en este momento se daría un intercambio entre dos formas diferentes de representación de la realidad. La situación se complejiza cuando la comunidad Sorda no vive aislada en un determinado territorio en relación con la cultura oyente y se ve obligada a intercambios constantes entre las representaciones comunicativas que los oyentes se hacen de los sujetos Sordos, en los intercambios comunicativos que deben ser mediados por la lengua y que tienen lugar dentro y fuera de la escuela. 
Es así como actividades difundidas universalmente, como ver televisión, cobran otro significado cuando lo hace una persona Sorda [sin intérprete o close caption]; lo mismo pasa con el baile cuando lo que se despliega en el sujeto Sordo es la vibración del sonido y no su escucha. Las actividades en sí mismas no cambian, lo que se modifica son las significaciones que se construyen alrededor de su práctica.

Por otra parte, la responsabilidad de la comunicación suele recaer en el Sordo, en un proceso de asimilación pura, en que "él" tiene que ajustarse a la mayoría dominante, negando la cultura Sorda, en la forma de una utopía que se vende a la persona sorda y su familia, que para incluirse debe ser y comportarse como algo que biológicamente no es, como un oyente, y pasarse a esta otra cultura para ser legitimado.

La interculturalidad ocurrirá realmente cuando la construcción del sujeto Sordo se concrete desde la lengua de señas que habla, según el territorio donde la aprendió y la carga cultural que recibe con ella, así como por la que recibe de los oyentes que habitan ese mismo espacio. Si entendemos que la mayoría de los estudiantes Sordos conoce la lengua de señas cuando ingresa al colegio, y si la institución promueve el acercamiento a la asociación y la comunidad Sorda, él mismo sabrá de estas en etapas tempranas; en caso contrario, la conocerá en edad adulta, así se encuentre en procesos de oralización.

En esta etapa escolar, los docentes y co-docentes deben fomentar y permitir un espacio en el aula para propiciar la sensibilidad intercultural en construcción de una cultura Sorda y una cultura Oyente.

\subsection{PRINCIPIOS DE EDUCACIÓN INTERCULTURAL}

En el desarrollo e implementación de la educación en general y su anexo en educación especial, el concepto de diversidad apunta a la multiculturalidad y no a la interculturalidad (Dietz, 2003). En este sentido, el concepto de justicia frente a las desigualdades para interpretar las condiciones de desventaja que produce el sistema en el caso mexicano; es un abordaje que puede tener puntos de encuentro con los contextos de estudiantes Sordos, donde se podría hacer referencia a las injusticias producidas por las autoridades que representan al Estado, concretizadas en políticas educativas poco pertinentes para los Sordos (COMIE, 2013). 
Una mirada de justicia frente a los aprendizajes posibles en el contexto escolar podría reflejar un aspecto como lo es la importancia de continuar estudios sobre la lectura en Sordos, no como lo mínimo sino como lo máximo esperado del éxito escolar, de modo que el sistema educativo asuma los deberes y derechos inherentes a la particularidad lingüística, y así el docente tenga herramientas y estrategias visuales y kinestésicas dentro de su competencia lingüística en relación con la enseñanza de la lengua escrita con estudiantes Sordos.

La interculturalidad para Sordos en virtud de la diferencia supone que el antónimo de diferencia no sea igualdad sino identidad. Es innegable que los cambios emergentes en la globalización y movilización de territorio originan planteamientos en principios de educación intercultural para desarrollar acciones entre una o dos culturas que comparten un espacio (Odina, 1999). Para la aplicación en materia educativa, estos referentes de interculturalidad implicarían un cambio en la forma como se concibe al Otro diverso, y en el caso que compete a escolares Sordos, se les dé pleno reconocimiento de su identidad, de modo que se proyecte en todos los órdenes de la estructura sociocultural y que no solo los dignifique en el ámbito educativo sino en el conjunto de la sociedad con sus prácticas y saberes de posibilidades interculturales que confluyen (Martínez, 2007).

En este sentido, se deben instalar en las agendas de educación las directrices de educación intercultural (Unesco, 2006), según el Principio: "La educación intercultural respeta la identidad cultural del educando impartiendo a todos una educación de calidad que se adecúe y adapte a su cultura". La interculturalidad se presenta entonces como una alternativa de atención que puede ser modificable, ya que es capaz de proponer cambios no solo en el sistema educativo, sino en la vida cotidiana de aquellos que interactúan en espacios educativos interculturales (Medina et al., 2009). Por ende, la interculturalidad reconoce al Otro como diferente y le permite la interacción en una relación simétrica, no lo borra ni lo aparta, sino que busca comprenderlo, dialogar con él y respetarlo.

La perspectiva de educación intercultural debe desarrollar acciones entre culturas que comparten un espacio (Odina, 1999), en las que se develen situaciones de carácter contextual, dialógica y situada con relación a la comunicación en la que convergen varios actores desde políticas de gobierno, formación y quehacer que evidencien la importancia de las prácticas docentes adecuadas para estudiantes Sordos. 
Los principios de educación intercultural, como plataforma de construcción de políticas públicas en educación especial que plantee tensiones entre los niveles de comunicación en lengua de señas chilena del estudiante Sordo y la responsabilidad lingüística y pedagógica que conlleva el ingreso a la escuela, debe propiciar relaciones comunicativas que integren de forma simétrica la lengua en contextos cotidianos mediados por intérpretes y favorezcan así el desarrollo del lenguaje.

De forma concreta, después de haber hecho un recorrido por situaciones comunicativas en la familia y contexto escolar, bilingüismo y modelos de enseñanza para Sordos, es claro que la escuela debe abordar de entrada la particularidad lingüística del estudiante (Carvajal et al., 2012) en oposición a un sistema educativo predominante, que pareciera no estar capacitado ni adaptado para afrontar el problema y que, más bien, evidencia las desigualdades socioculturales, supeditando a un estatus socioeconómico las mejores condiciones para el proceso educativo y más cuando se presentan barreras para la comunicación, como es el caso de los estudiantes Sordos. En particular, la escuela especial se propone desde un PEI bilingüe intercultural ${ }^{5}$, trascendiendo las relaciones étnicas y, por ende, lineamientos pedagógicos específicos, que deben considerarse en la evaluación desde el posicionamiento de la lengua de señas chilena.

En una educación intercultural y bilingüe (Rathmann et al., 2007) para estudiantes Sordos, se debe adecuar el entorno para propiciar el contacto con locutores nativos que favorezcan la adquisición natural. Desde el punto de vista pedagógico, este bilingüismo ha de ser construido, ya que no se adquiere de manera totalmente espontánea como podría pensarse en niños oyentes expuestos a dos lenguas. Es indispensable, en su lugar, que existan unas condiciones adecuadas para que el niño Sordo adquiera su primera lengua.

La educación intercultural en y con Sordos debe asumir entonces los deberes y derechos inherentes al sujeto Sordo en su particularidad lingüística (Indesor, 2014). Como se mencionó anteriormente, se requiere una propuesta que contemple una interculturalidad en Sordedad, no como derivación de pueblos originarios, sino como otra que represente la interacción en competencia

\footnotetext{
Es de importancia señalar que el concepto bilingüe para Sordos en este estudio se plantea con el objetivo de fortalecer la Lengua materna (L1) como la lengua de señas chilena y los componentes que se complementan y confrontan en el enfoque teórico-conceptual, desde la educación intercultural en Chile y las competencias comunicativas interculturales que subyacen dentro del aula.
} 
comunicativa intercultural con planes y programas propios, que propicien dinámicas simétricas de forma consensuada en el aula.

\section{DISCUSIÓN}

Abordar la interculturalidad implica una relación entre culturas y entre sociedades, entre el niño Sordo y la familia oyente. En esta etapa del desarrollo del infante, puede haber niveles de intercambio cultural, pero cuando el niño Sordo no ha desarrollado una lengua de señas ni identidad Sorda, no forma parte de la cultura Sorda y vive el proceso como infante o niño pequeño en una familia que tomará decisiones comunicativas para ejercer la asimilación a la lengua dominante, guiada hacia una oralización en la que simplemente tiene que hablar.

Como se ha dicho, el intercambio cultural se ha de dar con infantes Sordos que se encuentren en proceso de adquisición y desarrollo de la lengua de señas en relación con la familia oyente, para acentuar la interacción, la comunicación, la negociación y el enriquecimiento entre formas culturales diversas (Odina, 2005). Al negociar significados de comunicación en diada, se posibilitan diálogos simétricos donde prime el respeto y se legitime a la persona Sorda -al ponerse imaginativamente en su lugar- como un sujeto de deseos y necesidades, sin olvidar lo básico: que un infante Sordo, será un adulto Sordo.

Lo anterior insta a directivas, docentes y co-docentes en el colegio definir a qué tipo de interculturalidad están adscritos, siendo imprescindible la construcción de caminos interculturales desde la escuela en doble vía: la primera, que la institución promueva el acercamiento a la asociación de Sordos y la comunidad Sorda para que todos los estudiantes la conozcan e interactúen en etapas tempranas. Todo ello, sin borrar los antecedentes comunicativos en relaciones de país, institución, educación de formación.

Lo anterior insta, además, a una relación comunicativa orientada hacia la simetría en el punto de enunciación de la lengua de señas, asumida como lengua materna de un sujeto Sordo. En la institución, el docente o co-docente debe forjar y desarrollar en y por los Sordos una Sordedad activa, empírica, realizando aportes para el desarrollo de vocabulario pedagógico para la práctica docente en construcción de Otros caminos, y procesos de aprendizaje que guíen acciones visuales pertinentes para estudiantes Sordos en el aula. 
Como hipótesis implícita tendríamos que la sensibilidad del educador es un mediador del aprendizaje y que la sensibilidad intercultural es un concepto dinámico junto con las prácticas docentes, de modo que no basta el grado de conocimiento de la lengua del profesor y del estudiante en el espacio de la escuela.

Padres y cuidadores, antes del ingreso de los niños a la escuela y durante su permanencia en ella, usan la lengua de señas en el entorno cotidiano para situaciones básicas, y esta es asignada a la práctica docente como carga desbordante de responsabilidad lingüística (Fierro, 2003). Así, por ejemplo el docente entra a resolver situaciones de comportamiento, felicitaciones o castigos que los padres no asumieron en su momento en el hogar y para solucionarlas envían comunicados después del fin de semana para que la escuela, y específicamente el docente o co-docente, resuelvan por efectos de la comunicación.

Los hijos Sordos (con padres oyentes) (Burad y Humphries, 2010) sufren discriminación en el seno del hogar -audismo-, y en ocasiones llegan al aula frustrados y expresan comentarios como: "Mi mamá me habla, habla chino, no le entiendo".

Por lo anterior, la institución o escuela especial debe aportar a lo que antes denominamos responsabilidad lingüística docente, que se manifiesta cuando un educador sensible busca maneras de involucrar a todos y todas las personas del entorno, con un gran énfasis a la familia, y aborda situaciones en el aula de forma grupal e individual, que en su mayoría no son académicas sino familiares, como ocurre con las desavenencias o conflictos, en donde ejerce un rol protagónico, sin que supla el rol del padre o cuidador.

La importancia del desarrollo lingüístico de manera conjunta (familiar, estudiante, docente, institucional) para la sensibilidad intercultural en aspectos como los conceptos pedagógicos, constituye un desafío constante, dado que en todas las asignaturas falta vocabulario pertinente para posicionar la palabra/seña sola al azar, si no se trabaja antes la conceptualización de forma visual del término.

En esta correlación que se da en el aula, el docente fomenta acciones de grupo con el curso que favorecen la sensibilidad intercultural, con apoyo en las exigencias lingüísticas, y un vínculo comunicativo positivo, que promueve el aprendizaje, mediante preguntas que insten a respuestas asertivas y explo- 
ratorias, y brinden más oportunidades para el ensayo y error, pues equivocarse es parte del aprendizaje. De este modo, el docente desarrolla acciones para que el estudiante Sordo se anime a intentarlo de nuevo, permitiendo reconocer las reacciones y emociones que "gatilla" y encauzan la relación de acuerdo con las necesidades del mismo.

La plataforma para desarrollar o propiciar la sensibilidad intercultural, si bien parte del docente como sujeto, no se da en forma automática, sino que más bien es aprendida y perfectible, a partir de elementos como: leyes, recursos, familia, estudiantes y personal para potenciar comunicativa y pedagógicamente el encuentro en la negociación de dos culturas, con un Otro diverso en el aula.

Reconocer la presencia de los principios de educación intercultural en el discurso docente orientado hacia la educación de los Sordos en conocimientos, actitudes y competencias culturales, involucra una posición comunicativamente ética de parte de la institución, en función del respeto de la lengua que inste a buscar alternativas para conseguir una construcción conjunta en el desarrollo del lenguaje, con el fin de desarrollar una emoción positiva hacia la comprensión y apreciación de las diferencias culturales y promover la sensibilidad que requiere la competencia comunicativa intercultural (Baños, 2006, 2012).

Finalmente, para consolidar una educación intercultural que propenda hacia la negociación simétrica de las culturas en protagonismo, para reconocer al Otro diverso en prácticas docentes, para la selección comunicativa en el aula, la institución ha de orientar el camino hacia una educación bilingüe para Sordos. Por tal razón, debe gestar una posición con los organismos del gobierno que le ayuden a expandir la educación intercultural y que no se limite a los pueblos originarios, sino a las culturas y las lenguas. En este gradiente entramos todos y todas.

\section{AGRADECIMIENTOS:}

A la Comunidad Sorda de Chile.

Por sus valiosos aportes en el área; a las investigadoras: Adamo, Acuña, Lattapiat de la Universidad Metropolitana de Ciencias de la Educación-Chile.

Nota de autores: El presente trabajo de revisión teórica se encuentra adscrito al Programa de Doctorado de Ciencias de la Educación Mención Intercultural de la Universidad Santiago de Chile. 


\section{REFERENCIAS}

Adamo, D., Acuña, X. \& Cabrera I. (2009). Diccionario bilingüe lengua de señas chilena/español: Tomos I-II. Santiago de Chile: Universidad Metropolitana de Ciencias de la Educación.

Baños, R. (2006). La dimensión afectiva de la competencia comunicativa intercultural en la Educación Secundaria Obligatoria: Escala de Sensibilidad Intercultural. Revista de investigación educativa, 24(2), 353-372. Recuperado de http://revistas. um.es/rie/article/view/96891

Baños, R. (2012). No Verbales en la Comunicación Intercultural. Ra-Ximhai, 8(2). Disponible en http://www.redalyc.org/pdf/461/46123366010.pdf

Burad, V. \& Humphries, S. (2010). El audismo. Cultura Sorda [En línea]. Disponible en: http://www.cultura-sorda.eu/resources/Burad_Audismo-2010.pdf [Revisado el 20 de enero de 2012].

Carvajal, M., Tascon, T. \& Vásquez, S. (2012). Construyendo Caminos, Sordos y Oyentes pensando en la U. Santiago de Cali: Universidad del Valle.

CEPAL (2012). Comisión Económica para América Latina y el Caribe. Panorama Social de América Latina y el Caribe. Santiago de Chile: Ediciones CEPAL.

Chen, G. \& Starosta, W. (1998). A review of the concept of intercultural awareness. Disponible en http://digitalcommons.uri.edu/cgi/viewcontent.cgi?article $=1036 \&$ context $=$ com_facpubs

Chen, G. \& Starosta, W. (2000). Validity or a new instrument, the intercultural Sensitivity Scale (ISS) Foundations of Intercultural Communication. Disponible en http://digitalcommons.uri.edu/cgi/viewcontent.cgi?article=1019\&context=com_ facpubs

Chen, G. (1997). A Review of the Concept of Intercultural Sensitivity. Disponible en http://digitalcommons.uri.edu/cgi/viewcontent.cgi?article=1037\&context=com_ facpubs

COMIE (2013). El Consejo Mexicano de Investigación Educativa. Disponible en http:// www.comie.org.mx/v4/

Dietz, G. (2003). Multiculturalismo, interculturalidad y educación: una aproximación antropológica. España: Universidad de Granada. Disponible en http://www. scielo.org.mx/pdf/rms/v77n1/v77n1a8.pdf

Fierro, S. (2003). La práctica docente y sus dimensiones. España: Valorar-UG.

Hadjikakou, K., Christodoulou, D., Hadjidemetri, E., Konidari, M. \& Nicolaou, N. (2009). The experiences of Cypriot hearing adults with deaf parents in family, school, and society. Journal of deaf studies and deaf education, 14(4), 486-502. https://doi.org/10.1093/deafed/enp011

Indesor (2014). 10 años de Bilingüismo en Chile: Experiencias Pedagogías de la Escuela Intercultural Bilingüe para estudiantes Sordos. Chile: Editorial UMCE.

King, P. \& Baxter, M. (2005). A developmental model of intercultural maturity. Journal of college student development, 46(6), 571-592. Disponible en https://muse.jhu. edu/article/189017/pdf

Ladd, P. (2011). Comprendiendo la cultura sorda: en busca de la sordedad. Chile: Consejo Nacional de la Cultura y las Artes. Disponible en http://www.uchile.cl/ agenda/76733/presentacion-libro-comprendiendo-la-cultura-sorda 
Martínez, S. (2007). Bilingüismo, interculturalidad y educación, las comunidades indígenas y Sorda en Venezuela, una aproximación. Opción, 23(53). Disponible en http://www.scielo.org.ve/scielo.php?script=sci_arttext\&pid=S1012-1587200 7000200005

Massone, M., Buscaglia, V. \& Cvejanov, S. (2012). Estudios Multidisciplinarios sobre las Comunidades Sordas. Disponible en http://educacion.uncuyo.edu.ar/ nuevo-libro-estudios-multidisciplinarios-sobre-las-comunidades-sordas

Medina, A., Sevillano, M. \& De la Torre, S. (2009). Una universidad para el s. XXI. Espacio Europeo de Enseñanza Superior (EEES). Una mirada transdisciplinar, ecoformadora e intercultural. Mexico: CEPAL.

Mico-Cebrián, P. \& Cava, M. (2014). Sensibilidad intercultural, empatía, autoconcepto y satisfacción con la vida en alumnos de Educación Primaria. Infancia y Aprendizaje. Journal for the Study of Education and Development, 37(2), 342-363. https://doi.org/10.1080/02103702.2014.918819

Ministerio de Educación Chile. (2009). Ley 20.307/2009. Ley General de Educación. Chile: Biblioteca del Congreso Nacional de Chile.

Ministerio de Educación Chile. (2012). Lineamientos curriculares en competencia comunicativa, área lenguaje para 7 a 2 medio. Chile: Biblioteca del Congreso Nacional de Chile.

Morales, G., Ayala, A., Rivera, A. \& Sanhueza, R. (2017). Sensibilidad Intercultural: Diversidad Auditiva y Comunicación. Disponible en http://www.revistapasajes. com/gallery/3\%20oficial\%20articulo\%202017\%20enero\%20junio\%20\%20 pasajes\%20unam.pdf

Odina, T. (1999). La educación intercultural: concepto, paradigmas, realizaciones. Lectura de la pedagogía diferencial, 89-104. Disponible en http://www.lie.upn. $\mathrm{mx} /$ docs/Diplomados/Linealnter/Bloque3/Conceptuales/Lec4.pdf

Odina, T. (2005). La educación intercultural en la práctica escolar: investigación en el ámbito. Chile: CEPAL.

Rathmann, C., Mann, W. \& Morgan, G. (2007). Narrative structure and narrative development in deaf children. Deafness \& Education International, 9(4), 187-196. Disponible en http://onlinelibrary.wiley.com/doi/10.1002/dei.228/abstract

Schein, J. (1989). At home among strangers: Exploring the deaf community in the United States. USA: Gallaudet University Press.

Servicio Nacional de Discapacidad (Senadis). (2015). Segundo estudio Nacional de Discapacidad en Chile. Disponible en http://observatorio.ministeriodesarrollosocial.gob.cl/endisc/docs/Libro_Resultados_II_Estudio_Nacional_de_la_Discapacidad.pdf

Servicio Nacional de Discapacidad en Chile (Senadis). (2004). Primer estudio de discapacidad en Chile, pasos hacia un Modelo Integral del Funcionamiento Humano. Disponible en http://www.senadis.gob.cl/documentos/listado/137/estudios-estadisticas-informes

UNESCO (2006). Directrices para la Educación Intercultural Francia. Ginebra: Ediciones Unesco.

UNESCO (2010). Derechos de las minorías: Normas internacionales y orientaciones para su aplicación. Nueva York: Ediciones Unesco. 
Woodward, J. C. (1972). Implications for sociolinguistic research among the deaf. Sign Language Studies, 1(1), 1-7. Disponible en https://muse.jhu.edu/article/507135/ summary

Zemelman, H. (1992). Los horizontes de la razón: uso crítico de la teoría (Vol. 2). España: Anthropos Editorial.

Zemelman, H. (1998). Sujeto: existencia y potencia (Vol. 16). España: Anthropos Editorial.

Zemelman, H. (2010). Aspectos básicos en la propuesta de la conciencia histórica o del presente potencial. México: Ipecal. autor o el licenciante. 